\section{X-RAY ANALYSIS IN INDUSTRY}

$\mathrm{T}$ HE second X-ray conference of the Institute of Physics was held in Cambridge during April 9 and 10. Sir Lawrence Bragg was in the chair, and in his opening address he particularly welcomed the foreign men of science present, and warm applause followed his greeting to Prof. V. M. Goldschmidt.

In the first session the relative advantages of the powder method with cylindrical and flat specimens were discussed. Dr. A. J. Bradley pointed out the necessity for the accurate measurement of intensities of spectra for determining structures, for investigating superlattices, for examining strained crystals, and for finding temperature factors and atomic scattering curves. The practical difficulties are not great when the spectra are well separated, but when there are many lines close together there are difficulties in finding the background. Dr. Bradley showed some examples in which the background has to be estimated from the values on either side of a group of lines, and he described the design of the photometer with which the curves were obtained. The main theoretical difficulty is the correction for absorption, and for a cylindrical specimen the calculation of the correction is very complicated; Dr. Bradley gave an outline of the method by which he has solved the problem.

Dr. G. W. Brindley pointed out that the absorption correction is more easily dealt with if a flat specimen be used. Nevertheless, the method is not popular, because certain reflexions do not reach the film and the focusing is poor except on one part of the film. This latter property can, however, be used to increase the accuracy; by making the weakest line the sharpest, the peak intensities of the lines can be brought nearer together and so kept within the range of linear response of the film. Not only is the absorption correction simple, but also the absorption coefficient does not enter into the calculation of relative intensities.

Dr. Brindley described the construction of a flatspecimen camera designed to expose two specimens alternately, and explained how absolute intensities can be measured by means of a standard such as aluminium. He showed some results of measurements on potassium chloride, copper and cold-worked rhodium and copper.

Prof. J. M. Robertson spoke on the measurement of the intensities of spots on single-crystal photographs. $\mathrm{He}$ said that, while visual estimates of intensities can often be used to fix atomic parameters with surprising accuracy, it is sometimes important to obtain more exact data. He recommended the use of very small specimons, and the reduction of the background with moving-film cameras or by crystalreflected radiation. There is still difficulty in comparing intensities over a range of about $3,000: 1$, and the comparison of the $\beta$-spots of the strong reflexions with the $\alpha$-spots of the weaker ones can be used to overcome this. The presence of $\beta$-spots is, however, undesirable for other reasons, and alternatively one can use a shutter that allows the lower orders to be exposed for only a certain fraction of the total time of exposure. A still simpler method is to expose five films together in series so that each spot is recorded in five known ratios. The stronger spots on the last film can then be compared with the fainter ones on the first. Prof. Robertson gave some of the practical details to be taken into account in this method.
In the ensuing discussion, Dr. W. Hume-Rothery directed attention to the possibility of film shrinkage. during exposure, and said that in order to overcome it he finds it necessary to store his film at constant humidity and to maintain this humidity in the camera. Errors in lattice parameters can be overcome by fixing the films near the high-angle ends, but this may affect the measurement of the low orders. Mr. H. P. Rooksby and Dr. A. H. Jay recommended that the films should be stored in a lead-lined box near the X-ray tube, but Dr. HumeRothery said that he has not found this satisfactory.

In the evening, Mr. C. W. Bunn gave a lecture entitled "Towards Atomic Photography". He spoke on the problem of producing images of atoms. A magnification of at least $10^{2}$ would be required and the resolution would have to be great in proportion. Abbe's treatment of the resolving power of a microscope, however, leads to the conclusion that the waves used must be smaller than the details one wishes to see, and visible light is therefore much too coarse to detect atoms. X-rays have much smaller wave-lengths, but they cannot be effectively refracted in order to form an image. A beam of electrons, however, also has a short wave-length and can be refracted by electric or magnetic fields. This has led to the construction of the 'electron microscope', with which large molecules can be resolved. But this instrument is still far from being able to detect single atoms.

The formation of an image by optical methods can be resolved into the formation of a diffraction pattern, and the subsequent recombination of the diffraction spectra to form a final image. It is easy enough to get a diffraction pattern with $\mathrm{X}$-rays, but we cannot continue with the second process. It is possible, however, to collect the X-ray diffraction data and to perform the second process with light. Unfortunately, it is not possible to measure the phases of the X-ray spectra, and Mr. Bunn showed how this affects the results in the case of the image of a regular object in a microscope; as the microscope is racked back the image changes, but there is always some detail that might be mistaken for the real image. In some special cases the difficulty can be overcome, and Mr. Bunn showed a copy of Sir Lawrence Bragg's photograph of the atoms in diopside, $\mathrm{CaMg}\left(\mathrm{SiO}_{3}\right)_{2}$.

Mr. Bunn also showed some further developments of the application of optical methods to structure analysis, such as the determination of structure amplitudes by what he termed the 'fly's eye' method. A regular array of pin-hole images is formed photographically ; the diffraction pattern of the structure to be examined then gives a complete zone of spectra, and this may be compared with the observed X-ray spectra. In a similar way we can find the effects of faults in a structure when this cannot be done by calculation.

The third session began with a discussion of the proposal to change $\mathrm{X}$-ray wave-lengths to absolute values. Dr. H. Lipson explained that the current Angstrøm unit is now known to be in error by 0.2 per cent and suggested that it should be corrected. $\mathrm{He}$ read a letter from Prof. M. Siegbahn [see p. 502] who suggested that the question be deferred until the error has been determined more accurately. This seemed to be the general view, and Mr. Rooksby suggested that a change should not be made without international agreement on the conversion factor. Sir Lawrence Bragg therefore proposed that the 
question be raised with the American Society for $\mathrm{X}$ Ray and Electron Diffraction, and this was agreed to.

Then followed a series of papers on recent developments. Dr. A. H. Jay explained the significance of the formation of superlattices in alloys, and explained how they can be detected by X-rays. He stated that the formation cannot be regarded as a phase change because there is no radical change in structure.

Dr. Kathleen Lonsdale, who spoke on "Thermal Effects and Allied Phenomena", showed some slides illustrating the decrease in intensity of the main reflexions with rise of temperature, and the corresponding increase in intensity of the 'diffuse' reflexions. These reflexions may be regarded as arising in the following way. X-rays have such a high frequency that from their point of view all the atoms are stationary, although owing to thermal motion they may be displaced from their mean positions. Each of the elastic waves in a crystal can be regarded as producing a superlattice, and it is the combination of large numbers of superlattice reflexions that produces the diffuse reflexions.

Dr. Lonsdale emphasized the usefulness of the concept of reciprocal space for the study of this effect in particular and crystal structures in general, and showed how beautifully the fine detail can be explained by the theory. Jahn's formula for the isodiffusion surfaces has been verified experimentally on lithium, sodium, lead and tungsten single crystals, and it may soon be possible to find the elastic constants of a substance by this method.

Dr. Lipson introduced the subject of "Side-Band Formation" by saying that it is an example of a phenomenon for which the theory had been worked out many years previously to its discovery. In brief, the side-bands are essentially the same as one of Dr. Lonsdale's elastic waves 'frozen in', but in this case the wave is due to the incipient separation-in an alloy of copper, iron and nickel-of two phases similar in structure to the parent phase, but with different lattice parameters. Dr. Lipson also showed how the positions of the side-bands can be satisfactorily accounted for in reciprocal space, and that the wave-length and direction of the periodic variation can be found in this way. The intensities do not agree with the theory, however, without a correction for extinction; he thinks that the extinction effect was not given sufficient prominence during the first day of the conference.

Dr. Lipson then showed some results obtained by Dr. Vera Daniel on the decomposition of an alloy with time, and said that entirely new information about phase changes is being obtained.

In the discussion, Sir Lawrence Bragg remarked that the structures that give the side-bands are promising objects for the electron microscope. Mr. K. Hoselitz pointed out that the information is just that needed for the correlation of coercivity and erystal structure, and Dr. Lipson agreed that it would be useful to make the necessary magnetic measurements. Mr. H. J. Goldschmidt said that he had observed a similar phenomenon in certain mag. netic alloys.

Prof. Goldschmidt spoke of photographs of radioactive deposits that have been disintegrating for about a million years, and said that they show imperfections because of the recoil of the uranium atoms. Dr. P. P. Ewald directed attention to the similarity of Dr. Lonsdale's work to the examination of the diffraction of light waves by a crystal carrying ultra-sonic waves. He also asked about the effect of a periodic variation of the structural parameters of a crystal, but Dr. Lonsdale said that such effects would appear too far from the reciprocal lattice points to be easily detected. The question of whether superlattice formation should be included on equilibrium diagrams was raised by many speakers ; Sir Lawrence Bragg gave his opinion that it is not necessary to discuss whether the formation is a phase change or not, as now that we know the exact nature of the change it does not matter what name is given to it.

The last part of this session was devoted to a report on the preparation of the X-ray index proposed at the meeting held at Cambridge last year. Dr. A. J. C. Wilson presented the report; he gave a brief history of the index and explained the exact nature of the collaboration between the various bodies concerned.

At the opening of the final session, Dr. C. Sykes reported a resolution by the organizing committee that an X-Ray Crystallography Group of the Institute of Physics be formed. After assurances that the group would welcome chemists and others and was only to be regarded as a temporary measure, the resolution was carried.

The main subject of the final session-“"Quantitative Treatment of Line Broadening"-was introduced by Dr. F. W. Jones. He showed that the problem of estimating line breadths is complicated by the broadening due to other causes such as the divergence of the X-ray beam and the geometry of the camera. He explained his method of using a standard substance which gives no diffraction broadening, so that, by suitable treatment, this part of the broadening can be allowed for in the broadened lines. He pointed out that this method is unreliable when the diffrac. tion broadening is small compared with the geometrical broadening.

Dr. Wilson spoke on "The Effects of Distortion and other Imperfections". Among the possible causes of line broadening he listed small particle size, heat motion, 'mistakes', and distortion due to cold work. The broadening due to heat motion is different in appearance from the others; Dr. Lonsdale's diffuse reflexions are a manifestation of it. 'Mistakes' are errors in the building up of a lattice, and have been found in hexagonal cobalt, chrysotile, and $\mathrm{AuCu}_{3}$. The theory of mechanical distortion is far from complete, and on account of the difficulties, which Dr. Jones mentioned, of estimating the actual diffraction broadening of the spectra, it has not been found possible to settle unequivocally whether the broadening due to cold work is primarily due to fragmentation or to variation of lattice parameter. The experimental evidence tends towards the latter, and the differences between the various spectra can be satisfactorily accounted for in terms of elastic anisotropy.

In the discussion, Dr. Brindley pointed out that his results are in agreement with this view, and said also that the amount of extinction present in some practical cases cannot be accounted for if the crystals are very small. Dr. W. A. Wood, however, presented some new results which, he claimed, support his theory of fragmentation. On the other hand, Dr. E. Orowan showed a photograph from a bent wire, and another from the wire when it had been straightened, and said that these led to the other conclusion.

This discussion, on the whole, provided the best illustration of how such conferences help in allowing the exponents of different theories to state their views to each other, and in bringing their differences into 
relief so that the points to be settled can be more clearly seen.

An exhibition of apparatus and films was arranged by Dr. Wilson with the main purpose of illustrating points in the lectures. For example, the Cambridge Instrument Company showed photographs of its latest types of microphotometer, and Dr. Bradley's laboratory produced a set of powder cameras the diameters of which vary from $2.5 \mathrm{~cm}$. to $35 \mathrm{~cm}$.- a graphic demonstration of the way in which this type of camera has developed in the last twenty years.

Mr. Bunn showed some photographs illustrating the method of evaluating structure-amplitudes optically. Sir Lawrence Bragg's 'X-ray microscope', which may be said to have heralded this tendency to return to optical principles for solving crystal structures, was also on view, and was used to show diffraction by a three-dimensional grating as well as several of the effects described in Mr. Bunn's lectire.

In connexion with the papers on "Recent Developments", photographs from superlattices were exhibited; Dr. Lonsdale showed a beautiful series of prints of her 'diffuse reflexions', and Dr. Vera Daniel showed some of the photographs with which the 'side-band' effects have been studied.

The discussion on "Line Broadening" was illustrated by an exhibit by Mr. A. R. Stokes showing the broadening caused in the several ways enumerated by Dr. Wilson. In addition, Dr. D. P. Riley produced a series of photographs of coals taken with crystalreflected radiation. These photographs show a spreading of the central beam-'low-angle scattering' - that can be correlated with the actual particle-size in the specimen, as distinct from the crystal size that most X-ray measurements produce.

There were several other exhibits of general and metallurgical interest, and as a practical example of the working of an X-ray laboratory the Crystallographic Laboratory of the University was also open for inspection.

\section{BRITISH ELECTRICAL AND ALLIED INDUSTRIES RESEARCH ASSOCIATION}

T HE twenty-second annual report (E.R.A./T.331) of the British Electrical and Allied Industries Research Association summarizes the work which has been carried out during the year ended September 30,1942 , and, like its predecessor, lists by titles the various research reports which have been issued during the period. The work is reviewed under eighteen major classifications, among which, as was pointed out on the previous occasion, are again the important ones of dielectrics, cables and overhead lines, electric control apparatus, steam-power plant and condensers, magnetic materials, transformers, surge phenomena and rural electrification.

The report shows that the work of the Association is now carried on by 112 technical sections, subcommittees and panels comprised of technicians and scientific workers engaged in industry and in universities and other training institutions. Fiftyseven technical reports on a variety of subjects have been issued by the Association during the year, and fifty-one others were in an advanced stage of preparation at the end of the year. These may be divided as follows, giving, in each instance, first new reports and, secondly, reports in preparation: di- electrics, 20, 15; cables and overhead lines, 8, 4; electric control apparatus, 15, 16; steam-power plant and condensers, 2,2 ; communication interference, 3, 2 ; magnetic materials, 2, 2 ; transformers, 0,1 ; surge phenomena, 4, 3; safety problems, 0,3 ; rural electrification, 1,1 ; transformer noise, $2,2$.

The Information Bureau has been particularly active during the year, and Government and Service Departments are now making increasing use of its services. The Association's card index now contains nearly 30,000 special references, and current matter is being added at a substantial rate. The first half of the complete analytical index to the contents of E.R.A. reports, covering electrotechnical materials, has now been completed. Close co-operation has been effected with the British Standards Institution in connexion with the electrical engineering section of the Universal Decimal Classification and with the Insti. tution of Electrical Engineers in connexion with Science Abstracts.

The work of the Association has outgrown the capacity of the Perivale Laboratory in spite of the large amount of work which is still carried out by other bodies under its auspices, and this now necessitates removal of the laboratory to another site.

The E.R.A. is largely employed in finding specific answers to clearly formulated questions, and the former are of immediate value. Even more important contributions are being made by the E.R.A. in those fields in which the need for guidance is foreseen but the questions cannot yet be clearly put. It may take the industry many years to absorb a result of researches of the latter type, because this may involve a minor revolution in outlook and practice. Certain researches in hand fall definitely into this category; for example, some of those concerned with the phenomena of arcing, dielectric phenomena, properties of circuits, interference with communication circuits, surge phenomena, transformer design, magnetic materials and surface tension.

Notwithstanding the pressure of direct war work, serious fundamental research on dielectrics has been maintained. Industry in war-time has co-operated with and utilized the E.R.A. in dealing with many immediate problems affecting the successful utilization of particular insulating materials.

Of prime interest to the electricity supply industry are the long-range researches designed to increase the reliability, efficiency and safety, and heighten the performance, of plant used for the generation and distribution of electric power. The larger part of the work done falls within this category, and is linked closely with the user interest. Work on problems of electricity supply technology has been brought into increased activity and, in general, co-operation with authorjzed undertakings has been increased.

The investigation of breakdowns of plant and the devising of steps for prevention of the recurrence are the responsibility of engineers immediately concerned, but in so far as they generally involve surge phenomena, phenomena of arcing and questions of the properties of circuits, members can consult the E.R.A. Such work is not only of benefit to the member concerned but also benefits others similarly situated, and assists the investigators in establishing a true perspective as to the needs of the industry.

Continuous progress has been made in establishing liaison with all concerned with post-war reconstruction in the field of agriculture, and there has been increased appreciation of the contributions that the E.R.A. is making. 